\title{
One-stage revision of infected hip arthroplasty: outcome of 39 consecutive hips
}

\author{
Thomas Ilchmann $^{1}$ - Werner Zimmerli ${ }^{2}$ Peter Emil Ochsner ${ }^{3}$ - Bernhard Kessler ${ }^{2}$. \\ Lukas Zwicky $^{4} \cdot$ Peter Graber $^{2} \cdot$ Martin Clauss $^{3}$
}

Received: 13 March 2015 / Accepted: 19 May 2015 /Published online: 30 July 2015

(C) The Author(s) 2015. This article is published with open access at Springerlink.com

\begin{abstract}
Purpose There are various options for treating periprosthetic joint infection (PJI). Two-stage exchange has traditionally been the gold standard. However, if the appropriate surgical intervention is chosen according to a rational algorithm, the outcome is similar when using all types of interventions. In an observational cohort study, the outcome of patients with PJI after hip replacement treated with one-stage revision was analysed.

Methods All patients fulfilling all criteria for one-stage exchange according to the Infectious Diseases Society of America (IDSA) guidelines and six without preoperative identification of a microorganism were included. Implant removal, debridement and cemented or uncemented reimplantations were performed in a single intervention. If a cemented device was implanted, commercially available gentamicin cement was used in all cases. Antibiotic treatment was administered intravenously for at least 2 weeks, followed by oral therapy for a total duration of 3 months. Patients had standardised clinical and radiological follow-up visits.
\end{abstract}

Thomas Ilchmann

thomas.ilchmann@hirslanden.ch

$1 \quad$ Hirslanden Klink Birshof, Münchenstein, Switzerland

2 Interdisciplinary Unit for Orthopaedic Infections and Basel University Medical Clinic, Kantonsspital Baselland Liestal, Liestal, Switzerland

3 Interdisciplinary Unit for Orthopaedic Infections and Clinic for Orthopaedics and Trauma Surgery, Kantonsspital Baselland Liestal, Liestal, Switzerland

4 Clinic for Orthopaedics and Trauma Surgery, Kantonsspital Baselland Liestal, Liestal, Switzerland
Results Between 1996 and 2011, 38 patients (39 hips) were treated with a one-stage procedure and followed for at least 2 years. Coagulase-negative staphylococci were the most frequent pathogens, and polymicrobial infection was observed in five cases. In 25 hips, an uncemented revision stem was implanted, and 37 hips received an acetabular reinforcement ring. The mean follow-up was $6.6(2.0-15.1)$ years. No patient had persistent, recurrent or new infection. There were four stem revisions for aseptic loosening. The mean Harris Hip Score was 81 points (26-99) at the final follow-up.

Conclusions Excellent cure rate and function seen in our study suggest that one-stage exchange is a safe procedure, even without local antibiotic treatment, provided that the patient has no sinus tract or severe soft tissue damage, no major bone grafting is required and the microorganism is susceptible to orally administered agents with high bioavailability.

Keywords Hip arthroplasty $\cdot$ Infection · One-stage $\cdot$ Hip replacement $\cdot$ Treatment $\cdot$ Uncemented

\section{Introduction}

Periprosthetic joint infection (PJI) is a major complication after total joint replacement and causes additional surgery, functional impairment and high medical costs. There is an increasing incidence of PJI due to an increasing number of operations and patients at risk living with orthopaedic implants [1]. The diagnostic and therapeutic management of PJI is demanding and expensive. Various treatment options are discussed controversially, and reported case series are difficult to compare with each other. Prospective randomised trials are difficult or even impossible to achieve, and therefore lacking [2]. 
Two-stage exchange is an established and reliable treatment strategy for infection eradication [3] but is a complex and extensive surgical procedure. It requires at least two major surgical interventions, may cause loss of function due to softtissue and bone damage and is associated with considerable mortality $[2,3]$. One-stage exchange with the use of antibiotic-loaded cement has a reported success rate of up to $82 \%[4,5]$, but is inferior to two-stage exchange, if it is performed in a patient not qualifying for this procedure [6-9].

In aseptic stem revisions, uncemented stems have shown reliable long-term results [10]. For PJI, uncemented reimplantations are performed in two-stage exchanges [11, 12]. However, successful one-stage exchange with the use of uncemented implants has also been described [13-15]. At our hospital, an algorithm to stratify patients with established PJI to the most appropriate management was developed in the 1990s. All patients in this series were treated accordingly [7, 16]. These treatment concepts were adopted in the recent Infectious Diseases Society of America (IDSA) guidelines [6]. The different surgical treatment options are debridement and implant retention, one-stage exchange, two-stage exchange, resection arthroplasty or suppressive therapy.

In this retrospective cohort study, we assessed whether onestage exchange is a safe procedure in patients with PJI provided that they qualify according to the IDSA guidelines, except for preoperative isolation of a microorganism [6].

\section{Methods}

All patients with infectious symptoms after hip replacement (pain, local inflammation, fever) were evaluated for potential PJI. Patient's history and clinical findings were assessed, including previous interventions, symptom duration, antibiotic treatments and comorbidity. Standardised radiographs (pelvis anteroposterior and false profile) were taken and compared with all available previous radiographs to assess radiographic stability and bone loss. Laboratory analyses included white blood cell counts and C-reactive protein (CRP). In case of fever, blood cultures were drawn. Preoperative joint aspiration was routinely performed. Cell counts and differential of aspirated synovial fluid was determined [17]. Tissue samples and/ or synovial fluid were incubated for a prolonged period ( 2 weeks) to detect slow-growing microorganisms [18].

PJI was diagnosed according to IDSA guidelines. This definition includes signs and symptoms of local infection plus at least one of the following criteria: (1) growth of the same microorganism in at least two cultures of synovial fluid, periprosthetic tissue and/or sonication fluid; (2) visible pus surrounding the joint without other explanation (e.g. no crystals); or (3) acute inflammation on histopathological examination ( $>5$ neutrophils/high-power field). All patients fulfilled the modified criteria for one-stage exchange as defined by a published algorithm and IDSA guidelines [6, 7]: intact soft tissue (no sinus tract or abscess formation), susceptibility of causative microorganisms to antibiotics with activity against adhering pathogens (rifampin for staphylococci and fluoroquinolones for Gram-negative bacilli) [19]. In 6/39 hips, the microorganism was unknown at the time of surgery. However, all qualified for the microbiological requirements after surgery. These hips had low-grade infection and were treated with vancomycin plus amoxicillin/clavulanic acid until the microorganism and its susceptibility were known.

Revisions were performed via a transfemoral approach in 21 hips and a lateral approach in 18 . At least four to six biopsies were taken intraoperatively for culture and histology to confirm microbiological diagnosis, drug susceptibility and signs of inflammation. Debridement consisted in thorough removal of infected soft tissue, without bone resection. The operative site was repeatedly manually rinsed with $0.2 \%$ polyhexanide solution (Lavasept ${ }^{\circledR}$ ) with a syringe. Before reimplantation, the surgeon's gloves but not drapes or instruments were changed.

Most acetabular reconstructions (29 hips) were performed with a Müller acetabular reinforcement ring (Zimmer, Winterthur, Switzerland), as the implant is proven to be reliable and is frequently used at our hospital. In case of major defects (eight hips), we used a Burch Schneider antiprotrusio cage (Zimmer) combined with a cemented Müller low-profile polyethylene (PE) cup (Zimmer) [20]. Two reimplantations were made with uncemented press-fit cups (Müller SL and Allofit, both Zimmer). In the early cases, cemented stems were preferably used in the more elderly patients; in 13 hips, a cemented stem was implanted (12 Virtec, one $\mathrm{CDH}$, both Zimmer) - nine through a lateral approach. In later cases, uncemented fixation was preferred. Twenty-six uncemented stems were implanted (14 Wagner SL revision, 12 Revitan, both Zimmer) - 17 through a transfemoral approach, which was preferred in case of extensive bone cement distal to the tip of infected cemented stems and for uncemented stem fixation. In 15 hips, morselised bone grafts were used to reconstruct acetabular defects. Autologous (9), allogenous (2) and combined (4) bone grafts were used. In case of cemented fixation, standard gentamicin cement (Palacos R, later Palacos $\mathrm{R}+\mathrm{G}$, Heraeus Medical, Wehrheim, Germany) was used, irrespective of the susceptibility pattern of the microorganism, using a second-generation cementing technique. No additional antibiotics - either in cement or in bone graftswere administered. Up to four drains were used and remained in place for 3-5 days. In case of imminent fluid retention after drain removal, operative revision, including a second placement of drains, was performed.

In 20 hips, antibiotic treatment was started after arthrocentesis but before revision surgery. In the other 19 hips, it was started intraoperatively either empirically or according 
to culture results from synovial fluid. It was continued intravenously for a minimum of 2 weeks. In staphylococcal infections, rifampin ( $450 \mathrm{mg}$ twice daily per os) was added as soon as the wound was dry. After 2 weeks, antibiotics were switched to an oral regimen using drugs to which the infecting agent was susceptible and had excellent bioavailability (Table 1). The total duration of antibiotic treatment was 3 months. All complications and reoperations for any reason were recorded.

All patients had a prospective clinical and radiological follow-up during antibiotic therapy and after 1,2 and 5 years. Blood samples (blood cell counts, CRP) and standardised radiographs were taken. Subsidence, osteolysis and radiolucent lines were assessed. Functional results were documented with a modified Harris Hip Score (HHS).

\section{Results}

From 1996 to 2011, 39 patients with 40 hips underwent onestage exchange due to PJI. One patient died 2 months after revision surgery due to a carcinoma without any signs of persistence of infection. Due to the short follow-up, this case was excluded from the analysis. All others reached at least the 2year control, and no patient was lost to follow-up, which was a mean of 6.6 years $(2.0-15.1)$.

The mean duration of symptoms prior to revision surgery was 9.7 months (1.2-33.2). Twenty of the 38 patients were men; mean time after primary operation was 3.9 years $(0.4$ 16.5). Eight hips were previously revised for various reasons, and one hip had previous surgery for infection (debridement with implant retention for PJI), which had failed.

In a previous study, microbiological cure rates and functional results of patients treated with implant exchange for established PJI between 1985 and 2004 at our institution were described [13]. Twenty of these hips with a one-stage procedure were included in the study presented here.

In six hips, preoperative aspiration either revealed no fluid or synovial culture was negative. None of these patients had a history of previous antibiotic treatment. In these patients, causative bacteria were identified in blood cultures or intraoperative tissue biopsies. In three hips, synovial fluid was positive, but additional bacteria [coagulase-negative staphylococci (CNS) in all three cases $\}$ were detected from intraoperative biopsy samples. Table 1 shows microbiology and antimicrobial therapy from each of the 39 hip revisions. CNS were the most frequent pathogens $(n=20)$, followed by streptococci $(n=9)$, Staphylococcus aureus $(n=8)$, Gram-negative bacilli $(n=4)$, and Propionibacterium acnes $(n=3)$. Five episodes $(12.5 \%)$ were polymicrobial. In all patients undergoing empirical therapy, antibiotics were streamlined as soon as the susceptibility testing was available. In patients with staphylococcal infection, the mean time between surgery and administration of rifampin was 8 days (1-20). In six patients, evacuation of fluid retention was required during index hospitalisation, namely, 12-24 days after surgery. One patient with dementia had recurrent dislocations, which were treated with closed reduction. There were no other early reinterventions. No patient had a relapse or a new infection, and all were free of infection at the final follow-up visit. In four hips, a stem revision was performed: one uncemented stem 8 months after revision for early subsidence, and three cemented stems for symptomatic aseptic loosening after 27, 34 and 59 months; all had a complete radiolucent line (Fig. 1). In all four patients, microbiological healing was confirmed with negative biopsy cultures at revision.

Besides the revised stem, no uncemented stem showed subsidence $>5 \mathrm{~mm}$. None of the ten unrevised cemented stems had osteolysis or a complete radiolucent line, but six had an incomplete radiolucent line mainly in the proximal third. Mean HHS at the 2-year follow-up was 81 points (26-99).

\section{Discussion}

In PJI management, the most important aim is infection eradication. In our series, infection was controlled in all 39 hips. This excellent result was attained on the first attempt, except in six patients in whom surgical evacuation of postoperative fluid retention was performed during the index hospitalisation. In $90 \%$ of patients, the implant was still functional and in place at the last follow-up visit. Four stems were replaced because of loosening, which was not caused by low-grade infection, as confirmed with negative intraoperative cultures.

All patients had a proven PJI that did not qualify for debridement with implant retention [7]. In the same period of time, 79 patients a a two-stage exchange, as they were not appropriate for one-stage exchange [6]. In $>80 \%$ of cases, the infecting agent and its susceptibility were known before surgery. However, in six cases with suspected PJI, either arthrocentesis was not performed, there was no synovial fluid or synovia showed no bacterial growth (Table 1). In these cases, PJI was confirmed with positive intraoperative biopsy cultures. According to IDSA guidelines, these patients would not qualify for one-stage exchange. However, Choi et al. [21] showed that two-stage revision is not mandatory for all patients with no known preoperative microbiology. Nevertheless, in patients with unknown microbiology, the risk for failure is increased, since they may have PJI caused by difficult-to-treat microorganisms, such as small-colony variants [22]. In our series, no patient without preoperative culture results had a difficult-to-treat microorganism (Table 1).

Excellent treatment results in our study with a $100 \%$ infection cure rate may be because all patients had fair skin and soft tissue, good bone stock and microorganisms susceptible to an antibiotic with excellent bioavailability and efficacy on 
Table 1 Microorganisms and antimicrobial therapy

\begin{tabular}{|c|c|c|c|c|c|}
\hline No. & $\begin{array}{l}\text { Microorganism(s) } \\
\text { in synovia }\end{array}$ & $\begin{array}{l}\text { Microorganism(s) } \\
\text { in intraoperative biopsies }\end{array}$ & Antibiotic(s) IV & Antibiotic(s) orally & $\begin{array}{l}\text { Antibiotic } \\
\text { pretreatment }^{\text {a }}\end{array}$ \\
\hline 1 & CNS & $\mathrm{CNS}$ & Cefazolin & Ciprofloxacin/rifampin & Yes \\
\hline 2 & Enterobacter cloacae & $\begin{array}{l}\text { S aureus and } \\
\quad \text { Enterobacter cloacae }\end{array}$ & Piperacillin/tazobactam & Ciprofloxacin/rifampin & Yes \\
\hline 3 & $\mathrm{CNS}$ & $\mathrm{CNS}$ & Flucloxacillin & Ciprofloxacin/rifampin & Yes \\
\hline 4 & $\mathrm{No}^{\mathrm{c}}$ & CNS & Flucloxacillin & Ciprofloxacin/rifampin & No \\
\hline 5 & $\mathrm{No}^{\mathrm{c}}$ & $\mathrm{CNS}$ & Vancomycin/teicoplanin & Ciprofloxacin/rifampin & No \\
\hline 6 & $\mathrm{No}^{\mathrm{c}}$ & $\mathrm{CNS}$ & Flucloxacillin & Ciprofloxacin/rifampin & No \\
\hline 7 & Staphylococcus aureus & S. aureus/CNS & Flucloxacillin & Ciprofloxacin/rifampin & No \\
\hline 8 & Streptococcus sanguis & Streptococcus sanguis & Penicillin & Amoxicillin/rifampin & No \\
\hline 9 & $\mathrm{CNS}$ & $\mathrm{CNS}$ & Flucloxacillin & Ciprofloxacin/rifampin & Yes \\
\hline 10 & S. aureus & S. aureus & Flucloxacillin & Ciprofloxacin/rifampin & Yes \\
\hline 11 & CNS & CNS & Teicoplanin & Fusidic acid/rifampin & Yes \\
\hline 12 & Escherichia coli & E. coli & Amoxicillin/clavulanic acid & Ciprofloxacin & Yes \\
\hline 13 & Streptococcus agalactiae & Streptococcus agalactiae & Amoxicillin & Amoxicillin & Yes \\
\hline $14^{\mathrm{b}}$ & S. aureus & S. aureus & Flucloxacillin & Levofloxacin/rifampin & Yes \\
\hline $15^{\mathrm{b}}$ & S. aureus & S. aureus & Flucloxacillin & Levofloxacin/rifampin & Yes \\
\hline 16 & Viridans streptococci & Viridans streptococci & Penicillin & Levofloxacin/rifampin & Yes \\
\hline 17 & $\mathrm{CNS}$ & CNS & Flucloxacillin & Levofloxacin/rifampin & No \\
\hline 18 & Streptococcus milleri & Streptococcus milleri & Penicillin & Amoxicillin & Yes \\
\hline 19 & $\mathrm{No}^{\mathrm{d}}$ & Anaerobic streptococci & Amoxicillin/clavulanic acid & Clindamycin/rifampin & No \\
\hline 20 & S. aureus & S. aureus/CNS & Teicoplanin & Levofloxacin/rifampin & Yes \\
\hline 21 & $\mathrm{No}^{\mathrm{d}}$ & P. acnes/CNS & Penicillin & Clindamycin/rifampin & No \\
\hline 22 & $\mathrm{CNS}$ & CNS & Vancomycin & Levofloxacin/rifampin & No \\
\hline 23 & $\mathrm{CNS}$ & $\mathrm{CNS}$ & mycin & Minocycline & No \\
\hline 24 & $\mathrm{CNS}$ & $\mathrm{CNS}$ & Vancomycin & Fusidic acid/rifampin & Yes \\
\hline 25 & $\mathrm{CNS}$ & $\mathrm{CNS}$ & Vancomycin & Fusidic acid/rifampin & No \\
\hline 26 & Streptococcus mitis & Streptococcus mitis & Penicillin & Amoxicillin/rifampin & Yes \\
\hline 27 & Viridans streptococci & Viridans streptococci & Vancomycin & Fusidic acid/rifampin & No \\
\hline 28 & Viridans streptococci & Viridans streptococci & Penicillin & Amoxicillin/rifampin & No \\
\hline 29 & E. coli & CNS & Ceftriaxon & Ciprofloxacin & No \\
\hline 30 & $\mathrm{No}^{\mathrm{c}}$ & E coli & $\begin{array}{l}\text { Vancomycin/ } \\
\text { Amoxicillin/clavulanic acid }\end{array}$ & Ciprofloxacin & No \\
\hline 31 & E. coli & E. coli & Ceftriaxon & Ciprofloxacin & No \\
\hline 32 & $S$ aureus & $S$ aureus & Flucloxacillin & Levofloxacin/rifampin & Yes \\
\hline 33 & $S$ aureus & $S$ aureus & Penicillin & Levofloxacin/rifampin & Yes \\
\hline 34 & $\mathrm{CNS}$ & CNS & Vancomycin & Fusidic acid/rifampin & No \\
\hline 35 & $\mathrm{CNS}$ & $\mathrm{CNS}$ & Vancomycin & Fusidic acid/rifampin & Yes \\
\hline 36 & $\mathrm{CNS}$ & $\mathrm{CNS}$ & Vancomycin & Minocycline/rifampin & Yes \\
\hline 37 & $\mathrm{CNS}$ and $P$. acnes & $\begin{array}{l}\text { CNS and Propionibacterium } \\
\text { acnes }\end{array}$ & Penicillin & Clindamycin/rifampin & No \\
\hline 38 & Streptococcus mitis & Streptococcus mitis & Penicillin & Cefuroxime & Yes \\
\hline 39 & P. acnes & P. acnes & Amoxicillin/clavulanic acid & Clindamycin/rifampin & No \\
\hline
\end{tabular}

CNS coagulase-negative staphylococci

a Antibiotic pretreatment after arthrocentesis, before one-stage exchange

b Same patient treated on both sides

c Arthrocentesis without fluid aspiration

${ }^{\mathrm{d}}$ No growth in synovial fluid 

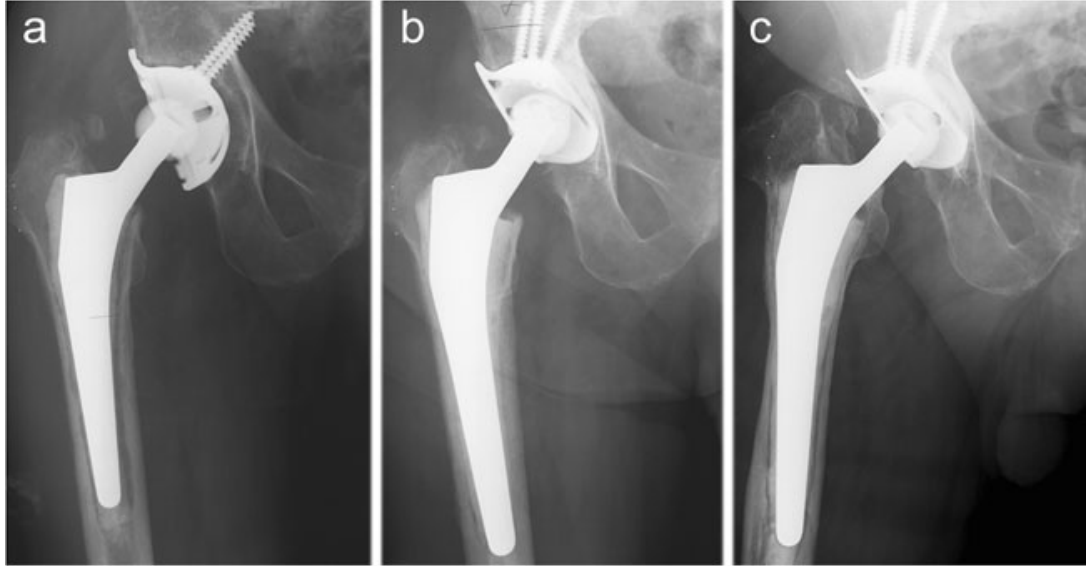

Fig. 1 A 78-year-old male patient with primary hip replacement due to osteoarthritis. He developed symptoms over a period of 4 months. Ten months postoperatively, periprosthetic joint infection (PJI) was diagnosed, and puncture revealed Streptococcus sanguis. A one-stage exchange was performed using a Müller reinforcement ring and a cemented stem. The infection was treated with penicillin IV for 2 weeks

biofilm bacteria. Cases with identified difficult-to-treat bacteria were stratified for two-stage procedures [22, 23], as were all cases with severe soft-tissue destruction, since the risk for failure with direct exchange is increased in these patients. In staphylococcal infections, a rifampin-containing regimen, and in Gram-negative infections, fluoroquinolone, was administered [19]. Rifampin treatment was delayed until the wound was dry in order to avoid emergence of resistance [24].

Preservation of function is the second most important aim in treating patients with PJI. However, only limited data are reported regarding joint function after treatment of infected hip arthroplasty [13]. The extent of surgical debridement is difficult to quantify; however, a strategy with direct exchange, including radical debridement, of any tissue disregarding possible loss of function should be avoided [5]. Our aim was to preserve functionally important structures and to perform moderate debridement excluding extensive bone and softtissue resection. As previously shown, HHS in patients with one-stage exchange for PJI is comparable with that in patients treated with revision for aseptic loosening and similar to that is a series of primary implantations [13].

For reimplantation, we used the same established implant systems, with documented long-term results, as we used in the routine for aseptic loosening [10]. In our case series, three of 13 cemented stems failed due to symptomatic aseptic loosening after a mean of 40 months. Thus, uncemented reimplantation might be the method of choice even in case of infection $[11,25]$. On the acetabular site, we mainly used reinforcement rings due to modular options for restoring the hip centre; there were no re-revisions for cup loosening. Other uncemented revision cups might be used as well. With the described strategy, a long-lasting fixation was achieved, which did not differ from revision operations without infection [20]. and amoxicillin/rifampin per os for 3 months. Over time, the patient experienced lateral hip pain, and the stem was re-revised for aseptic loosening. a) Anteroposterior (AP) radiograph before one-stage exchange. b AP radiograph directly after one-stage exchange of both components. c AP radiograph 34 months after one-stage exchange showing a complete radiolucent line of the stem before re-revision

Drains were used to avoid dead spaces and to dry out the wound as soon as possible, which was a prerequisite for starting rifampin therapy [24]. For that reason, fluid retention was surgically removed in six patients. As no local antibiotics were administered, this should not affect the concentration of antibiotics in the operative field.

Standard gentamicin-loaded bone cement was used to fix the cup in the rings and the cemented stems. In 26 hips with uncemented femoral components, the use of cement was limited to PE cup fixation in the acetabular ring. It might be debated that local antibiotic release contributed to curing the infection.

A limitation of our observational cohort study is the absence of a control group. For stratification to a one-stage exchange, we used criteria based on a well-established algorithm and IDSA guidelines, except for preoperative knowledge of the microorganism [6, 7]. Since patients without synovial fluid (punctio sicca) or with negative preoperative culture without synovial cell counts in favour of infection [6] could also have aseptic loosening, one-stage exchange is indicated. In view of these treatment guidelines, we consider randomisation to different treatment strategies as nonethical, since each patient should be treated with the least invasive procedure for which he or she qualifies [7]. Therefore, randomisation in two groups with one- or two-stage procedures could hardly be justified.

The strength of our study is the standardised inclusion according to accepted criteria $[6,7]$, the prospective acquisition of standardised data in a cohort database and management of each patient with a dedicated specialised team. In addition, reporting the use of uncemented stems for one-stage revision in patients with PJI adds new knowledge. It illustrates that local antibiotics are not indispensable for infection elimination during direct exchange. However, this observation must be confirmed in larger studies. 
In conclusion, one-stage revision is an attractive and safe option for treating selected patients with PJI, and PJI can be cured even without local administration of antibiotics.

Acknowledgments We thank Susanna Häfliger for following all patients and collecting data of the register.

Open Access This article is distributed under the terms of the Creative Commons Attribution 4.0 International License (http:// creativecommons.org/licenses/by/4.0/), which permits unrestricted use, distribution, and reproduction in any medium, provided you give appropriate credit to the original author(s) and the source, provide a link to the Creative Commons license, and indicate if changes were made.

\section{References}

1. Dale H, Fenstad AM, Hallan G, Havelin LI, Furnes O, Overgaard S, Pedersen AB, Karrholm J, Garellick G, Pulkkinen P, Eskelinen A, Makela K, Engesaeter LB (2012) Increasing risk of prosthetic joint infection after total hip arthroplasty. Acta Orthopaedica 83:449458. doi:10.3109/17453674.2012.733918

2. Lange J, Troelsen A, Thomsen RW, Soballe K (2012) Chronic infections in hip arthroplasties: comparing risk of reinfection following one-stage and two-stage revision: a systematic review and meta-analysis. Clinical Epidemiology 4:57-73. doi:10.2147/CLEP.S29025

3. Berend KR, Lombardi AV Jr, Morris MJ, Bergeson AG, Adams JB, Sneller MA (2013) Two-stage treatment of hip periprosthetic joint infection is associated with a high rate of infection control but high mortality. Clinical Orthopaedics and Related Research 471:510 518. doi:10.1007/s11999-012-2595-X

4. Choi HR, Kwon YM, Freiberg AA, Malchau H (2013) Comparison of one-stage revision with antibiotic cement versus two-stage revision results for infected total hip arthroplasty. The Journal of Arthroplasty 28:66-70. doi:10.1016/j.arth.2013.02.037

5. Gehrke T, Kendoff D (2012) Peri-prosthetic hip infections: in favour of one-stage. Hip International : the Journal of Clinical and Experimental Research on Hip Pathology and Therapy 22(Suppl 8): S40-45. doi:10.5301/HIP.2012.9569

6. Osmon DR, Berbari EF, Berendt AR, Lew D, Zimmerli W, Steckelberg JM, Rao N, Hanssen A, Wilson WR, Infectious Diseases Society of A (2013) Executive summary: diagnosis and management of prosthetic joint infection: clinical practice guidelines by the Infectious Diseases Society of America. Clinical Infectious Diseases : an Official Publication of the Infectious Diseases Society of America 56:1-10. doi:10.1093/cid/cis966

7. Zimmerli W, Trampuz A, Ochsner PE (2004) Prosthetic-joint infections. The New England Journal of Medicine 351:1645-1654. doi: 10.1056/NEJMra040181

8. Gahlot N, Bahadur R (2014) Prosthetic joint infection following total hip replacement: results of one-stage versus two-stage exchange. International Orthopaedics 38:2427-2428. doi:10.1007/ s00264-014-2517-5

9. Wolf M, Clar H, Friesenbichler J, Schwantzer G, Bernhardt G, Gruber G, Glehr M, Leithner A, Sadoghi P (2014) Prosthetic joint infection following total hip replacement: results of one-stage versus two-stage exchange. International Orthopaedics 38:1363-1368. doi:10.1007/s00264-014-2309-y

10. Regis D, Sandri A, Bonetti I, Braggion M, Bartolozzi P (2011) Femoral revision with the Wagner tapered stem: a ten- to 15 -year follow-up study. The Journal of Bone and Joint Surgery British volume 93:1320-1326. doi:10.1302/0301-620X.93B10.25927
11. Fink B, Grossmann A, Fuerst M, Schafer P, Frommelt L (2009) Two-stage cementless revision of infected hip endoprostheses. Clinical Orthopaedics and Related Research 467:1848-1858. doi: 10.1007/s11999-008-0611-y

12. Masri BA, Panagiotopoulos KP, Greidanus NV, Garbuz DS, Duncan CP (2007) Cementless two-stage exchange arthroplasty for infection after total hip arthroplasty. The Journal of Arthroplasty 22:72-78. doi:10.1016/j.arth.2006.02.156

13. De Man FH, Sendi P, Zimmerli W, Maurer TB, Ochsner PE, Ilchmann T (2011) Infectiological, functional, and radiographic outcome after revision for prosthetic hip infection according to a strict algorithm. Acta Orthopaedica 82:27-34. doi:10.3109/ 17453674.2010 .548025

14. Zeller V, Lhotellier L, Marmor S, Leclerc P, Krain A, Graff W, Ducroquet F, Biau D, Leonard P, Desplaces N, Mamoudy P (2014) One-stage exchange arthroplasty for chronic periprosthetic hip infection: results of a large prospective cohort study. The Journal of Bone and Joint Surgery American volume 96, e1. doi: 10.2106/JBJS.L.01451

15. Hansen E, Tetreault M, Zmistowski B, Della Valle CJ, Parvizi J, Haddad FS, Hozack WJ (2013) Outcome of one-stage cementless exchange for acute postoperative periprosthetic hip infection. Clinical Orthopaedics and Related Research 471:3214-3222. doi: 10.1007/s11999-013-3079-3

16. Giulieri SG, Graber P, Ochsner PE, Zimmerli W (2004) Management of infection associated with total hip arthroplasty according to a treatment algorithm. Infection 32:222-228. doi:10. 1007/s15010-004-4020-1

17. Schinsky MF, Della Valle CJ, Sporer SM, Paprosky WG (2008) Perioperative testing for joint infection in patients undergoing revision total hip arthroplasty. The Journal of Bone and Joint Surgery American volume 90:1869-1875. doi:10.2106/JBJS.G.01255

18. Zappe B, Graf S, Ochsner PE, Zimmerli W, Sendi P (2008) Propionibacterium spp. in prosthetic joint infections: a diagnostic challenge. Archives of Orthopaedic and Trauma Surgery 128: 1039-1046. doi:10.1007/s00402-007-0454-0

19. Zimmerli W, Widmer AF, Blatter M, Frei R, Ochsner PE (1998) Role of rifampin for treatment of orthopedic implant-related staphylococcal infections: a randomized controlled trial. Foreign-Body Infection (FBI) Study Group. JAMA : the Journal of the American Medical Association 279:1537-1541

20. Ilchmann T, Gelzer JP, Winter E, Weise K (2006) Acetabular reconstruction with the Burch-Schneider ring: an EBRA analysis of 40 cup revisions. Acta Orthopaedica 77:79-86. doi:10.1080/ 17453670610045722

21. Choi HR, Kwon YM, Freiberg AA, Nelson SB, Malchau H (2013) Periprosthetic joint infection with negative culture results: clinical characteristics and treatment outcome. The Journal of Arthroplasty 28:899-903. doi:10.1016/j.arth.2012.10.022

22. Sendi P, Rohrbach M, Graber P, Frei R, Ochsner PE, Zimmerli W (2006) Staphylococcus aureus small colony variants in prosthetic joint infection. Clinical Infectious Diseases : an Official Publication of the Infectious Diseases Society of America 43:961-967. doi:10.1086/507633

23. Rasouli MR, Tripathi MS, Kenyon R, Wetters N, Della Valle CJ, Parvizi J (2012) Low rate of infection control in enterococcal periprosthetic joint infections. Clinical Orthopaedics and Related Research 470:2708-2716. doi:10.1007/s11999-012-2374-8

24. Achermann Y, Eigenmann K, Ledergerber B, Derksen L, Rafeiner P, Clauss M, Nuesch R, Zellweger C, Vogt M, Zimmerli W (2013) Factors associated with rifampin resistance in staphylococcal periprosthetic joint infections (PJI): a matched case-control study. Infection 41:431-437. doi:10.1007/s15010-012-0325-7

25. Bori G, Munoz-Mahamud E, Cune J, Gallart X, Fuster D, Soriano A (2014) One-stage revision arthroplasty using cementless stem for infected hip arthroplasties. The Journal of Arthroplasty 29:10761081. doi:10.1016/j.arth.2013.11.005 